\title{
On the Equivalence of Two Singular Matrix Pencils
}

\author{
By J. Williamson, The Johns Hopkins University.
}

(Received 18th October, 1935. Read 1st November, 1935.)

In a recent paper ${ }^{1}$ Turnbull, discussing a rational method for the reduction of a singular matrix pencil to canonical form, has shown how the lowest row, or column, minimal index may be determined directly without reducing the pencil to canonical form. It is the purpose of this note to show how all such indices may be determined, and at the same time to give conditions, somewhat simpler than the usual ones, for the equivalence of two matrix pencils.

For simplicity we adopt, as far as possible, Turnbull's notation and, for convenience of the reader, in $\S 1$ give an abstract of the first part of his paper.

1. Let

$$
\Lambda=r A+s B=\left(r a_{i j}+s b_{i j}\right), \quad i=1,2, \ldots, n ; j=1,2, \ldots, n^{\prime}
$$

be a matrix pencil, where the elements $a_{i j}, b_{i j}$ all belong to the field $K$, while $r$ and $s$ are independent variables. We exclude the case in which $A$ is a scalar multiple of $B$. If $P$ and $Q$ are two non-singular matrices, with elements in $K$, of orders $n$ and $n^{\prime}$ respectively, such that

$$
P \Lambda Q=\Lambda_{1}=r C+s D,
$$

the pencils $\Lambda$ and $\Lambda_{1}$ are said to be equivalent in $K$ or briefly " to be equivalent."

Let $\rho$ be the rank of $\Lambda$ in $r$ and $s$; i.e. every determinant of order $\rho+1$ of $\Lambda$ vanishes identically in $r$ and $s$ while at least one determinant of order $\rho$ does not. If $\mu=n-\rho$ and $\mu^{\prime}=n^{\prime}-\rho$, then $\mu \geqq 0$ and $\mu^{\prime} \geqq 0$. If one of the integers $\mu$ or $\mu^{\prime}$, say $\mu>0$, the pencil (1) is singular. By an application of Smith's Theorem²,

'H. W. Turnbull, "On the reduction of singular matrix pencils," Proc. Edin. Mieth. Soc., 4 (1935)), 67.

2 Turnbull \& Aitken, Canonical Matrices, p. 23. 
Turnbull shows that the rows of $\Lambda$ satisfy exactly $\mu$ independent relations of the type

$$
\sum_{i=1}^{n} \phi_{i} \text { row }_{i}=0
$$

where $\phi_{i}$ is a polynomial in $r$ and $s$ with coefficients in $K$.

The relation (3) may be written in the form of a vector equation,

$$
\theta \Lambda=\left[\phi_{1}, \phi_{2}, \ldots, \phi_{n}\right](r A+s B)=0,
$$

identically in $r$ and $s$. The $\mu$ vectors $\theta$ are independent in the sense that they satisfy no homogeneous linear equation with coefficients, which are polynomials in $r$ and $s$. We may further suppose that the components $\phi_{i}$ of $\theta$ are homogeneous polynomials in $r$ and $s$ of the same degree and that they have no common factor. We thus have $\mu$ vectors $\theta_{i}, i=1,2, \ldots, \mu$, which annihilate $\Lambda$, that is satisfy the equation

$$
\theta_{i} \Lambda=0, \quad i=1,2, \ldots, \mu .
$$

We arrange the vectors $\theta_{i}$ in ascending degree so that, if $\theta_{i}$ is of degree $m_{i}$,

$$
0 \leqq m_{1} \leqq m_{2} \leqq, \ldots, \leqq m_{\mu} .
$$

This set of integers (6) is the set of Kronecker minimal indices of row dependence characterising the singular pencil $\Lambda$. A like set $\left\{m^{\prime}{ }_{i}\right\}, \mu^{\prime}$ in number, refers to column dependence and is obtained by considering the column vectors $\psi$, which satisfy the equation $\Lambda \psi=0$. These sets $\left\{m_{i}\right\}$ and $\left\{m^{\prime}{ }_{i}\right\}$ are invariant under transformations of type (2) and, together with the invariant factors, completely characterise the pencil $\Lambda$. Let

$$
\begin{aligned}
M_{1}=\left[\begin{array}{ll}
A & B
\end{array}\right], & M_{2}=\left[\begin{array}{lll}
A & B & 0 \\
0 & A & B
\end{array}\right], & M_{3}=\left[\begin{array}{llll}
A & B & 0 & 0 \\
0 & A & B & 0 \\
0 & 0 & A & B
\end{array}\right], \ldots \\
N_{1}=\left[\begin{array}{l}
A \\
B
\end{array}\right], & N_{2}=\left[\begin{array}{ll}
A & 0 \\
B & A \\
0 & B
\end{array}\right], & N_{3}=\left[\begin{array}{ccc}
A & 0 & 0 \\
B & A & 0 \\
0 & B & A \\
0 & 0 & B
\end{array}\right], \ldots
\end{aligned}
$$

so that $M_{i}$ is a matrix of in rows and $(i+1) n^{\prime}$ columns and $N_{i}$ a matrix of $(i+1) n$ rows and $i n^{\prime}$ columns. Let $\rho_{i}$ and $\rho^{\prime}{ }_{i}$ denote the ranks of $M_{i}$ and $N_{i}$ respectively. Then the integers

$$
\mu_{i}=i n-\rho_{i}, \quad \mu_{i}^{\prime}=i n^{\prime}-\rho_{i}^{\prime}
$$

are all greater than or equal to zero. 
2. Let $V$ be a vector with elements in $K$ which annihilates $M_{k}$, i.e. which satisfies the equation

$$
V M_{k}=\mathbf{0} .
$$

The vector $V$ is of dimension $n k$ and may be written in the form

$$
V=\left(v_{1}, v_{2}, \ldots, v_{k}\right)
$$

where each component vector $v_{i}$ is of dimension $n$. It now follows that

$$
v_{1} A=0, \quad v_{k} B=0, \quad v_{i} B+v_{i+1} A=0, \quad i=1,2, \ldots, k-1 .
$$

As a consequence of (9) the vector

$$
\psi=v_{1} r^{k-1}+v_{2} r^{k-2} s+\ldots+v_{k} s^{k-1}
$$

satisfies $\psi(r A+s B)=0$ identically in $r$ and $s$. We say that $\psi$ is of order $k-1$ and corresponds to the vector $V$. If $\psi$ can be written as a linear combination of vectors of order $<k-1$, we say that $\psi$ is reducible. If $\psi$ cannot be so expressed, we say that $\psi$ is irreducible. We now prove the lemma:

Lemma I. The vector $\psi$ in (10) corresponding to $V$ is reducible, if and only if the first component vector $v_{1}$ of $V$ is the first component of a vector $U$ which annihilates $M_{k-1}$.

Proof. Let the first component vector of $U$ be $v_{1}$. Then, if 0 denotes the zero vector of dimension $n$, the vector $V-(U, 0)=(0, W)$ annihilates $M_{k}$, so that $W$ annihilates $M_{k-1}$. If $\psi_{1}$ and $\psi_{2}$ are the vectors corresponding to $U$ and $W$ respectively,

$$
\psi=r \psi_{1}+s \psi_{2}, \text { and } \psi \text { is reducible. }
$$

Conversely, if $\psi$ is reducible, $\psi$ has the form (11), where $\psi_{1}$ and $\psi_{2}$ are of order $k-2$. If $U$ is the vector corresponding to $\psi_{1}$, which annihilates $M_{k-1}$, the first component of $V$ is the same as the first component of $U$ and our lemma is proved.

As in $\S 1$, by an application of Smith's theorem, there exist exactly $\mu_{k}$ linearly independent linear relations connecting the rows of $M_{k}$. That is, there exist exactly $\mu_{k}$ linearly independent vectors $V_{a}$ with elements in $K$, which annihilate $M_{k}$, i.e. which satisfy

$$
V_{a} M_{k}=0, \quad a=1,2, \ldots, \mu_{k} .
$$

Moreover, every vector, which annihilates $M_{k}$, is a linear combination of the vectors $V_{a}$ of (12), so that the vectors $V_{a}$ form a basis for the 
set of all vectors annihilating $M_{k}$. Each vector $V_{a}$ in (12) is of dimension $k n$ and may be written as

$$
V_{a}=\left[v_{a 1}, v_{a 2}, \ldots, v_{a k}\right],
$$

where each component vector $v_{a j}$ is of dimension $n$.

Let the vectors $V_{a}$ be so arranged that their first component vectors $v_{11}, v_{21}, v_{31}, \ldots, v_{\lambda_{k} 1}$, are linearly independent, but that

$$
v_{j 1}=\sum_{a=1}^{\lambda_{k}} c_{j a} v_{a 1}, \quad j=\lambda_{k}+1, \ldots, \mu_{k} \text {. }
$$

Now the vectors

$$
\begin{aligned}
U_{a} & =V_{a}, & a & =1,2, \ldots, \lambda_{k}, \\
U_{a} & =V_{a}-\sum_{\beta=1}^{\lambda_{k}} c_{a \beta} V_{\beta}, & a & =\lambda_{k}+1, \ldots, \mu_{k},
\end{aligned}
$$

are linearly independent and hence also form a basis for the set of vectors which annihilate $M_{k}$.

We may therefore suppose that the vectors $V_{a}$ of (12) are such that, as regards their first component vectors,

$$
v_{11}, v_{21}, \ldots, v_{\lambda_{k^{1}}} \text { are independent, } v_{a 1}=0, a>\lambda_{k} \text {. }
$$

If 0 denotes the zero vector of dimension $n$, each of the $\lambda_{k}$ vectors $W_{\beta}$ and the $\mu_{k}$ vectors $X_{a}$, defined by

$$
\begin{cases}W_{\beta}=\left(V_{\beta}, 0\right), & \beta=1,2, \ldots, \lambda_{k}, \\ X_{a}=\left(0, V_{a}\right), & a=1,2, \ldots, \mu_{k},\end{cases}
$$

is of dimension $(k+1) n$ and annihilates $M_{k+1}$.

Lemma 2. The $\mu_{k}+\lambda_{k}$ vectors (14) are linearly independent with respect to $K$.

Proof. Let

$$
\sum_{\beta=1}^{\lambda_{k}} c_{\beta} W_{\beta}+\sum_{a=1}^{\mu_{k}} \cdot d_{\alpha} X_{a}=0
$$

then, since the first component vector of $X_{a}$ is zero, $\sum_{\beta=1}^{\lambda_{k}} c_{\beta} v_{\beta 1}=0$, and by (13) $c_{\beta}=0, \beta=1,2, \ldots, \lambda_{k}$. Hence $\sum_{a=1}^{\mu_{k}} d_{a} X_{a}=0$ and consequently $\sum_{a=1}^{\mu_{k}} d_{a} V_{\alpha}=0$. As the $\mu_{k}$ vectors $V_{a}$ are linearly independent this last equation implies $d_{a}=0, a=1,2, \ldots, \mu_{k}$. Consequently the lemma is proved. 
But there exist $\mu_{k+1}$ linearly independent vectors which annihilate $M_{k+1}$. Accordingly a non-negative integer $\sigma_{k}$ must exist such that

$$
\mu_{k+1}=\mu_{k}+\lambda_{k}+\sigma_{k}, \quad \sigma_{k} \geqq 0 .
$$

Hence there also exist $\sigma_{k}$ vectors

$$
Y_{\gamma}=\left(y_{\gamma 1}, y_{\gamma 2}, \ldots, y_{\gamma, k+1}\right), \quad \gamma=1,2, \ldots, \sigma_{k},
$$

which annihilate $M_{k+1}$ and such that the $\mu_{k+1}$ vectors $W_{\beta}, X_{a}, Y_{y}$ are linearly independent. (If $\sigma_{k}=0$, no vector $Y_{\gamma}$ appears).

LEMMA 3. The first component vectors $v_{\beta 1}, y_{\gamma 1}$ of $W_{\beta}$ and $Y_{\gamma}$ respectively are linearly independent with respect to $K$, where $\beta=1,2, \ldots, \lambda_{k}$, $\gamma=1,2, \ldots, \sigma_{k}$.

Proof. Let

Then

$$
\sum_{\beta=1}^{\lambda_{k}} c_{\beta} v_{\beta 1}+\sum_{\gamma=1}^{\sigma_{k}} d_{\gamma} y_{\gamma 1}=0
$$

$$
\sum_{\beta=1}^{\lambda_{k}} c_{\beta} W_{\beta}+\sum_{\gamma=1}^{\sigma_{k}} d_{\gamma} Y_{\gamma}=(0, G)
$$

where, since the vector on the left of (17) annihilates $M_{k+1}$, the vector $G$ annihilates $M_{k}$. By there mark that follows (12), such a vector must take the form $G=\sum_{a=1}^{\mu_{k}} f_{a} V_{a}$, so that $(0, G)=\sum_{\alpha=1}^{\mu_{k}} f_{\alpha} X_{\alpha}$. Therefore, by (17),

$$
\sum_{\beta=1}^{\lambda_{k}} c_{\beta} W_{\beta}+\sum_{\gamma=1}^{\sigma_{k}} d_{\gamma} Y_{\gamma}-\sum_{\alpha=1}^{\mu_{k}} f_{a} X_{a}=0
$$

Since the vectors $W_{\beta}, X_{a}, Y_{\gamma}$ are linearly independent, $c_{\beta}=d_{\gamma}=f_{a}=0$, $\beta=1,2, \ldots, \lambda_{k} ; \gamma=1,2, \ldots, \sigma_{k} ; a=1,2, \ldots, \mu_{k}$. Accordingly the lemma is proved.

The formula corresponding to (15) when $k$ is replaced by $k+1$ is

$$
\mu_{k+2}=\mu_{k+1}+\lambda_{k+1}+\sigma_{k+1}, \sigma_{k+1} \geqq 0,
$$

where by definition $\lambda_{k+1}$ is the maximum number of vectors among those which annihilate $M_{k+1}$, whose first component vectors are linearly independent. But a vector $U$, which annihilates $M_{k+1}$, is a linear combination of the vectors $W_{\beta}, X_{a}, Y_{\gamma}$, and accordingly its first component vector $u_{1}$ is a linear combination of the vectors 
$v_{\beta 1}$ and $y_{\gamma 1}$, where $\beta=1,2, \ldots, \lambda_{k}, \gamma=1,2, \ldots, \sigma_{k}$. Since, by lemma 3 , these $\lambda_{k}+\sigma_{k}$ vectors are linearly independent, it follows that

$$
\lambda_{k+1}=\lambda_{k}+\sigma_{k}
$$

and accordingly (18) becomes

$$
\mu_{k+2}=\mu_{k+1}+\lambda_{k}+\sigma_{k}+\sigma_{k+1}
$$

so from (15) we have

$$
\sigma_{k+1}=\mu_{k+2}+\mu_{k}-2 \mu_{k+1}, \quad k=1,2, \ldots, .
$$

It is easily seen that formula (20) also holds for $k=-1,0$, if $\mu_{-1}$ and $\mu_{0}$ are defined to be zero.

The vectors $\psi_{y}, \gamma=1,2, \ldots, \sigma_{k}$, which correspond to the vectors $Y_{Y}$ in (16), annihilate $r A+s B$ and are of order $k$. As a consequence of lemma 3 , no linear combination of the vectors $y_{\gamma 1}$ is equal to a linear combination of the vectors $v_{\beta 1}$. Hence no linear combination of the first components of the vectors $Y_{\gamma}$ is equal to the first component of a vector which annihilates $M_{k}$. Consequently, by lemma 1, all linear combinations, with constant coefficients, of the $\sigma_{k}$ vectors $\psi_{\gamma}$ are irreducible. Moreover, if $\phi$ is any vector of order $k$ which annihilates $\Lambda$, and $U$ is the corresponding vector which annihilates $M_{k+1}$, then

$$
U=\sum_{\beta=1}^{\lambda_{k}} b_{\beta} W_{\beta}+\sum_{a=1}^{\mu_{k}} c_{a} X_{a}+\sum_{\gamma=1}^{\sigma_{k}} d_{\gamma} Y_{\gamma}
$$

It follows by an argument similar to the above that the vector $\phi-\sum_{\gamma=1}^{\sigma_{k}} d_{\gamma} \psi_{\gamma}$ is reducible. Hence the $\sigma_{k}$ vectors $\psi_{\gamma}$ form a maximal set of vectors of order $k$, with the property that no linear combination of them, with constant coefficients, is reducible. Consequently, the $\sigma_{k}$ vectors $\psi_{\gamma}$ form a maximal set of independent vectors $\theta$ satisfying (5) of index $k$. Accordingly, we have proved

Theorem I. The number of minimal row indices (6) which have the value $k$ is exactly $\mu_{k+1}+\mu_{k-1}-2 \mu_{k}$.

CoR. 1. If $\mu_{k+1}$ is the first of the sequence $\mu_{1}, \mu_{2}, \mu_{3}, \ldots$ which is different from 0 , then $k$ is the value of the smallest minimal index of row dependence and $\mu_{k+1}$ is the number of such indices which are equal. This is Turnbull's Theorem 2. 
Cor. 2. The number of minimal column indices (6) which have the value $k$ is exactly

$$
\mu^{\prime}{ }_{k+1}+\mu_{k-1}^{\prime}-2 \mu_{k}^{\prime} \text {. }
$$

By (20) and (8) we have

$$
\begin{aligned}
\sigma_{k+1} & =(k+2) n-\rho_{k+2}+k n-\rho_{k}-2(k+1) n+2 \rho_{k+1} \\
& =2 \rho_{k+1}-\rho_{k+2}-\rho_{k}
\end{aligned}
$$

and similarly the analogous formula

$$
\sigma_{k+1}^{\prime}=2 \rho_{k+1}^{\prime}-\rho^{\prime}{ }_{k+2}-\rho^{\prime}{ }_{k} .
$$

Conversely, the $\rho_{k}$ can now be expressed in terms of the $\sigma_{k}$. Accordingly we have proved

Cor. 3. The minimal indices $\left\{m_{i}\right\}$ and $\left\{m_{i}^{\prime}\right\}$ are completely determined by the ranks of the matrices $M_{k}$ and $N_{k}$. Conversely the ranks $\rho_{k}, \rho^{\prime}{ }_{k}$ of the matrices $M_{k}$ and $N_{k}$ are completely determined by the minimal indices $\left\{m_{i}\right\}$ and $\left\{m_{i}^{\prime}\right\}$.

In order to determine the minimal indices it is sufficient to form the sequence of the positive or zero integers

Since

$$
\begin{gathered}
\mu_{1}, \quad \mu_{2}-\mu_{1}, \quad \mu_{3}-\mu_{2}, \quad \mu_{4}-\mu_{3}, \ldots \\
\mu_{k+1}-\mu_{k}-\left(\mu_{k}-\mu_{k+1}\right)=\sigma_{k} \geqq 0,
\end{gathered}
$$

each term in the sequence is greater than or equal to its predecessor. The difference between the $(k+1)$ th term and the $k$ th term of the sequence $(21)$ is the number of minimal row indices which have the value $k$. Since

$$
\sigma_{0}+\sigma_{1}+\sigma_{2}+\ldots+\sigma_{k}=\mu_{k+1}-\mu_{k}
$$

the $(k+1)$ th term of $(21)$ gives the total number of independent vectors of order $\leqq k$, which annihilate $\Lambda$. But the maximum number of such vectors is $\rho$, where $\rho$ is the rank of $\Lambda$ in $r$ and $s$. Accordingly

$$
\mu_{k+1}-\mu_{k} \leqq \rho .
$$

Hence in forming the sequence $(21)$, if the $(k+1)$ th term has the value $\rho$ all succeeding terms will have the value $\rho$ and we need calculate no further.

By a proof exactly similar to that of Turnbull's Theorem 3, if $Y=\left(y_{1}, y_{2}, \ldots, y_{k+1}\right)$ is one of the vectors (16), the component vectors $y_{1}, y_{2}, \ldots, y_{k+1}$ are linearly independent. Since each $y_{i}$ is of dimension $n$, it follows that $k<n$ and consequently that

$$
\sigma_{k}=0, \quad k>n-1 \text {. }
$$


On the Equivalence of Two Singular Matrix Pencils 2.31

Hence in forming the sequence (21) we need never proceed beyond the $n$th term; and in corollary $3, k$ takes only the values $1,2, \ldots, n$ and $k^{\prime}$ the values $1,2, \ldots, n^{\prime}$.

Let $\Lambda_{1}=r C+s D$ be a second pencil of the same order as $\Lambda$ and let

$$
I_{k}, k=1,2, \ldots, n ; \quad J_{k^{\prime}}, k^{\prime}=1,2, \ldots, n^{\prime},
$$

be the matrices for this pencil corresponding to $M_{k}$ and $N_{k}$ respectively. Since a necessary and sufficient condition that the two pencils $\Lambda$ and $\Lambda_{1}$ be equivalent in the sense of (2), is that $\Lambda$ and $\Lambda_{1}$ have the same invariant factors and the same minimal indices ${ }^{1}$, as a consequence of corollary 3 we have

THEOREM 2. A necessary and sufficient condition that two pencils $r A+s B$ and $r C+s D$, with elements in a field $K$ be equivalent in $K$ is:

(a) that the invariant factors of $r A+s B$ be the same as the invariant factors of $r C+s D$,

(b) that the rank of $M_{k}$ be the same as the rank of $I_{k}, k=1,2, \ldots, n$,

(c) that the rank of $N_{k^{\prime}}$ be the same as the rank of $J_{k^{\prime}}, k^{\prime}=1,2, \ldots, n^{\prime}$.

1 Turnbull and Aitken, Op. cit., p. 129. 